\title{
User Software for Numerical Study of Josephson Junction with Magnetic Momenta
}

\author{
Pavlina Atanasova $^{1, \star}$, Stefani Panayotova ${ }^{1}$, Yury Shukrinov ${ }^{2,3}$, Ilhom Rahmonov ${ }^{2,4}$, and \\ Elena Zemlyanaya ${ }^{2,3}$ \\ ${ }^{1}$ University of Plovdiv Paisii Hilendarski, 24 Tzar Asen, 4000 Plovdiv, Bulgaria \\ ${ }^{2}$ Joint Institute of Nuclear Research, Dubna, Moscow Region, 141980, Russia \\ ${ }^{3}$ Dubna State University, Dubna, 141980, Russia \\ ${ }^{4}$ Umarov Physical Technical Institute, TAS, Dushanbe, 734063, Tajikistan
}

\begin{abstract}
A user software for numerical study of a Josephson junction model with magnetic momenta is presented. Computer implementation has been done by means of Wolfram Mathematica using the extensive capabilities of this system to create interactive dynamic objects. Two methods for numerical solution of the respective system of ordinary differential equations are implemented: the four-step Runge-Kutta algorithm and the Runge-Kutta-Fehlberg method with predetermined accuracy. Results of numerical simulation are presented to confirm the correctness of the calculations done with the developed software.
\end{abstract}

\section{Introduction}

Josephson junctions in combination with magnetic moments occupy an important place in "hot" fields of science like spintronics, nanotechnology, superconductivity, condensed matter physics, low temperature physics [1]. They represent interest in a number of practical applications and are subjects in many research areas [2]. Usually such problems cannot be solved analytically and the only way of investigation is by numerical studies. The numerical analysis of such multiparameter physical problems in a difficult undertaking. Thus, an important task is to create a suitable user software which implements the corresponding numerical methods as well as the visualization of the results. The chosen programming language for the creation of such software is Wolfram Mathematica [3] which is known as a finished product for the ultimate technical application and is convenient for research and experimentation.

In this paper we describe a user software for Josephson Junctions Simulations (USJJS) in the numerical study of the Josephson junctions with magnetic momenta. Using this software one can compute accurate values of some important physical parameters of the system under electric current pulse. We show that our method can demonstrate a magnetization reversal effect [4]. This effect is very important for superconducting spintronics and could be useful in the frame of applications in the quantum computing field [1].

\footnotetext{
^e-mail: atanasova@uni-plovdiv.bg
} 


\section{Mathematical model}

The geometry of the system of superconductor/ferromagnetic/superconductor Josephoson junction (SFS JJ) under consideration is presented in Fig. 1. The ferromagnetic easy-axis is directed along the $z$-axis, which is also the direction $n$ of the gradient of the spin-orbit interaction. The magnetization component $m_{y}$ is coupled with the Josephson current $I_{s}$, which is along the $x$-axis. The magnetic moment $\mathbf{M}$ is specified by its three spatial components along the axes. The dynamics of the magnetic

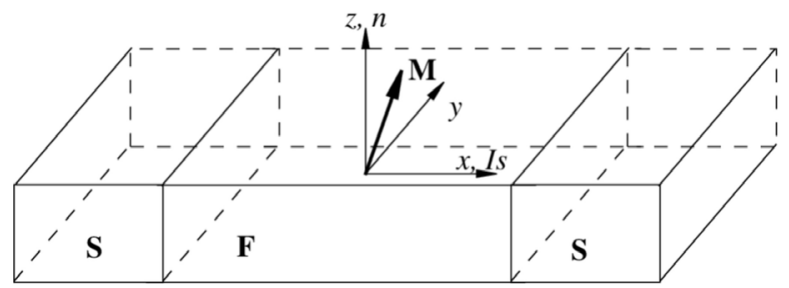

Figure 1. Geometry of the considered SFS JJ system. Here S is superconductor and F - ferromagnetic.

moment and phase difference in the SFS JJ is described by the following problem [4] of four ordinary first-order differential equations with initial conditions in dimensionless form:

$$
\begin{aligned}
& \frac{d m_{x}}{d t}=\frac{\omega_{F}}{1+\alpha^{2}}\left\{-m_{y} m_{z}+G r m_{z} \sin \left(\varphi-r m_{y}\right)-\alpha\left[m_{x} m_{z}^{2}-G r m_{x} m_{y} \sin \left(\varphi-r m_{y}\right)\right]\right\}, \\
& \frac{d m_{y}}{d t}=\frac{\omega_{F}}{1+\alpha^{2}}\left\{m_{x} m_{z}-\alpha\left[m_{y} m_{z}^{2}-G r\left(m_{z}^{2}+m_{x}^{2}\right) \sin \left(\varphi-r m_{y}\right)\right]\right\}, \\
& \frac{d m_{z}}{d t}=\frac{\omega_{F}}{1+\alpha^{2}}\left\{-G r m_{x} \sin \left(\varphi-r m_{y}\right)-\alpha\left[G r m_{y} m_{z} \sin \left(\varphi-r m_{y}\right)-m_{z}\left(m_{x}^{2}+m_{y}^{2}\right)\right]\right\}, \\
& \frac{d \varphi}{d t}=\frac{1}{w}\left(I_{\text {pulse }}(t)-\sin \left(\varphi-r m_{y}\right)\right), \quad I_{\text {pulse }}(t)= \begin{cases}A_{s}, & t \in\left[t_{0}-1 / 2 \Delta t, t_{0}+1 / 2 \Delta t\right] ; \\
0, & \text { otherwise, }\end{cases} \\
& m_{x}(0)=0, \quad m_{y}(0)=0, \quad m_{z}(0)=1, \quad \varphi(0)=0, \quad t \geq 0,
\end{aligned}
$$

where $\omega_{F}$ is the ferromagnetic resonance frequency, $\alpha$ is the damping parameter, $G$ is a parameter of the phase difference coupling, $r$ is the spin-orbit coupling parameter, $w=V_{F} /\left(I_{c} R\right)=\omega_{F} / \omega_{R}$, $V_{F}=\hbar \omega_{F} /(2 e), I_{c}$ is the critical current, $R$ is the resistance of $\mathrm{JJ}, \omega_{R}=2 e I_{c} R / \hbar$ is the characteristic frequency. The parameters of the amplitude $A_{s}$, the middle time of influence $t_{0}$ and the time interval of influence $\Delta t$ characterise the electric current pulse. The time dependent unknown functions are the magnetic moment components $m_{x}(t), m_{y}(t), m_{z}(t)$ and the phase difference $\varphi(t)$.

The superconducting current $I_{s}(t)$ is calculated via the function $\varphi(t)$ by the following formula $I_{s}(t)=I_{c} \sin \left(\varphi(t)-r m_{y}(t)\right), t \geq 0$.

\section{Numerical approach and user software USJJS}

The above system of ordinary differential equations with initial conditions was solved by the fourstep Runge-Kutta method and independently by the Runge-Kutta-Fehlberg method, which ensures a predetermined accuracy of the calculations. Several modules have been implemented for the visualisation of the results within of Wolfram Mathematica using the extensive capabilities of this system to 


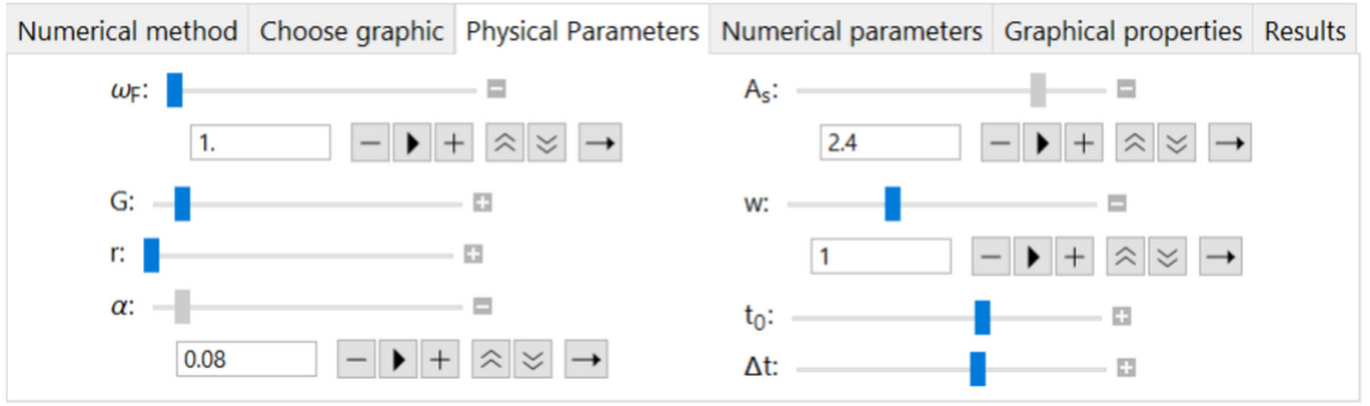

Figure 2. Menu of the USJJS where "Physical parameters" Tab is selected

create interactive dynamic objects. The results can be displayed in different ways depending on what is needed to the user.

The user software USJJS for the SFS JJ system study provides both graphical and tabular formatting options. Fig. 2 depicts how the USJJS menu looks. The menu is organized with the help of the following Tab Views: "Numerical method", "Choose Graphic", "Physical parameters", "Numerical parameters", "Graphical properties" and "Results". In "Numerical Method", the user can select one of two numerical simulation methods (the classical four-step Runge-Kutta algorithm or the RungeKutta-Fehlberg method). In "Choose Graphic", the user can choose one of nine types of graphics, each of them invokes a respective module that implements it. For the first eight types, each graph is displayed individually, and the ninth option "All Graphic" shows all graphs together. In the "Physical parameters" submenu (Fig. 2), specific parameter values can be entered and a change of function can be traced. In "Numerical parameters", the system's capabilities allow the manipulation of numerical parameters in an analogous way. Here, the "Animation Time Mode" Check Box is available to trace the dynamics of the functions. In the "Graphical properties" Tab, the user can "play" with the settings of the graphics. The last Tab "Results" propose a button that shows the user a table with all the numerical results of the calculations.

\section{Numerical results and conclusions}

To verify the correctness of the USJJS, we recovered all the numerical results from [4]. Excellent qualitative and quantitative agreement between our simulations and the simulations in [4] has been obtained. The instant in Fig. 3 reproduces the results in Fig. 2(b) of [4]. The parameters in this simulation are: $\omega_{F}=1, G=10, r=0.1, \alpha=0, A_{s}=1.5, w=1, t_{0}=25, \Delta t=6$.

To analyze the influence of the pulse electric current amplitude $A_{s}$, we make a simulation with the above values of parameters in the USJJS animation mode over the parameter $A_{s}$. One can observe how the function $m_{z}(t)$ is moving while the parameter $A_{s}$ is gradually growing. In the interval $A_{s} \in(0 ; 0.3]$, the current pulse changes the direction of the magnetic moment from up $\left(m_{z}=1\right)$ to down $\left(m_{z}=-1\right)$ only for a small time interval. After that the magnetic moment becomes again up to $m_{z}=1$ and stays that way. In the interval $A_{s} \in(0.3 ; 0.7]$, a gradual transition from upper direction of the magnetic moment to the down position is observed. Between values of $A_{s}=0.7$ and $A_{s}=0.9, m_{z}$ stays down to -1 . At the value of $A_{s}=1.2$, the magnetic moment again keeps stable its upper position $m_{z}=1$.

The magnetization reversal effect is demonstrated in Fig. 4 for the parameter values $\omega_{F}=1$, $G=50 \pi, r=0.1, \alpha=0.1, A_{s}=2.1, w=1, t_{0}=25, \Delta t=6$. On this figure the dynamics of the magnetic moment components, phase difference, phase diagrams $m_{z}-m_{x}, m_{z}-m_{y}, m_{y}-m_{x}$ and superconducting current are displayed. After the influence of signal in $t \in[22,28]$ the transition of 

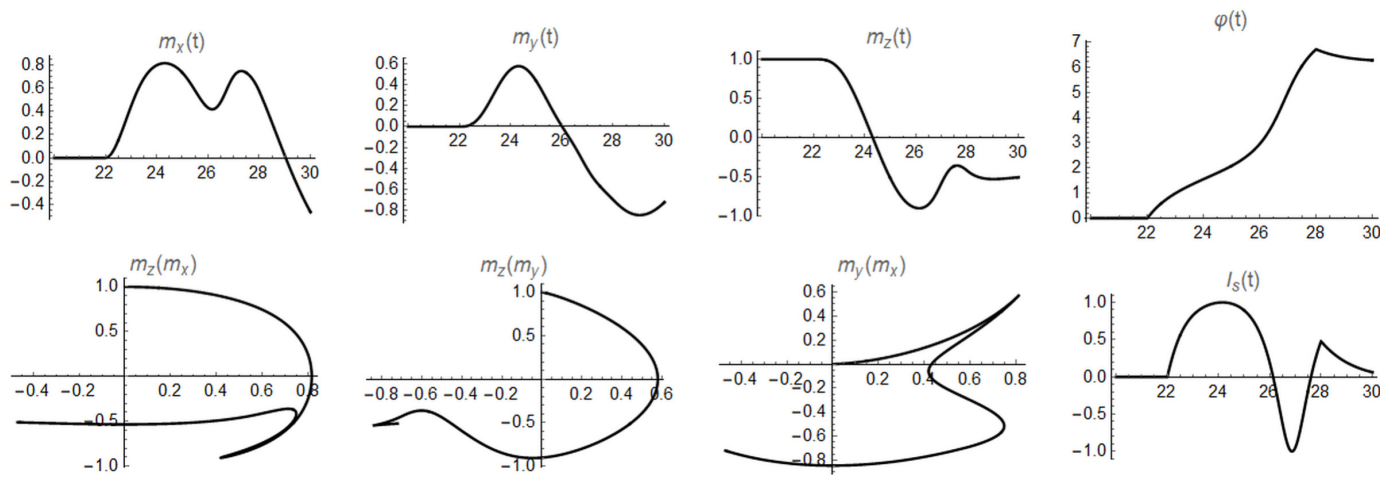

Figure 3. Results of the simulations with the same parameter values as in Fig. 2(b) of [4]
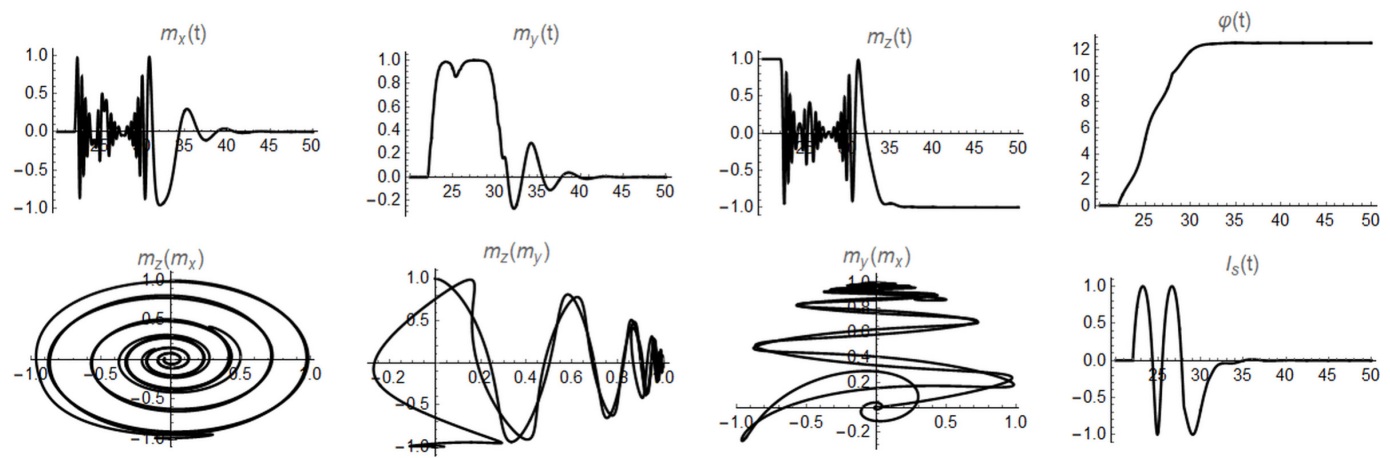

Figure 4. The magnetization reversal effect simulation in case of parameters $\omega_{F}=1, G=50 \pi, r=0.1, \alpha=0.1$, $A_{s}=2.1, w=1, t_{0}=25$

the $m_{z}$ component from the upper direction $m_{z}=1$ to the down one $m_{z}=-1$ is clearly seen while the components $m_{x}$ and $m_{y}$ come to zero.

An analogous investigation can be done with the help of our USJJS over the influence of the other physical parameters on the SFSJJ system behaviour. Thus, by means of the $S$, one can find accurate values of some important physical parameters of the electric current pulse and Josephson junction, in which the magnetization reversal effect is observed. This could be useful in the investigation of quantum computing models considering the two directions of magnetization as bits of information. Also, this effect could be evidenced in physical experiments.

\section{Acknowledgement.}

The work is supported by project FP17-FMI-008, Bulgaria, by the JINR-Bulgaria cooperation program, and by the RFBR (projects 15-29-01217, 16-52-45011, 17-01-00661).

\section{References}

[1] Jacob Linder and W.A.Jason Robinson, Nature Physics 11, 307 (2015)

[2] X.Waintal and P.W.Brouwer, Phys. Rev. B 65, 054407 (2002)

[3] http://reference.wolfram.com

[4] Yu.M.Shukrinov, I.R.Rahmonov, K.Sengupta, and A.Buzdin, Appl.Phys.Lett. 110, 182407 (2017) 